\title{
Construction of a new cervical anatomically adaptive titanium mesh cage based on measurements of cervical geometry: A morphological and cadaveric study
}

\author{
TENG LU ${ }^{1}$, ZHONGYANG GAO ${ }^{1}$, JIALIANG LI ${ }^{1}$, NING LIU ${ }^{2}$, YIBIN WANG ${ }^{1}, \mathrm{CHAO} \mathrm{LIU}^{3}$ and XIJING HE ${ }^{1}$ \\ ${ }^{1}$ Department of Orthopedics, Second Affiliated Hospital of Xi'an Jiaotong University, Xi'an, Shaanxi 710004; \\ ${ }^{2}$ Department of Spine Surgery, Hanzhong Central Hospital, Hanzhong, Shaanxi 723000; ${ }^{3}$ Department of Education, \\ Second Affiliated Hospital of Xi'an Jiaotong University, Xi'an, Shaanxi 710004, P.R. China
}

Received February 6, 2019; Accepted July 22, 2021

DOI: $10.3892 / \mathrm{etm} .2021 .10691$

\begin{abstract}
Mismatch between the titanium mesh cage and cervical geometries is an important factor that induces subsidence in anterior cervical corpectomy and fusion (ACCF). The aim of the present study was to construct a new quadrate anatomically adaptive titanium mesh cage (AA-TMC) that matches well with the cervical geometries and segmental alignment in one- and two-level ACCF. Computed tomography (CT) scans of 54 individuals were used to measure the cervical endplate geometries. X-rays of 74 young individuals were used to measure the intervertebral body angle (IBA) and intervertebral body height (IBH) of the surgical segments. The AA-TMC was designed based on these measured parameters. A total of 18 cervical cadaveric specimens underwent successive oneand two-level ACCF using the AA-TMC. Postoperatively, the specimens underwent $\mathrm{CT}$ scanning to assess the degree of matching of the TMC-endplate interface (TEI), IBA and IBH. A TEI interval $<0.5 \mathrm{~mm}$ was considered well matching. In the sagittal plane, $93.8 \%$ of the inferior endplates were arched, whereas $94.8 \%$ of the superior endplates were flat. In the coronal plane, $82.9 \%$ of the inferior endplates and $93.8 \%$ of the superior endplates were flat. A total of 91.7 and $94.4 \%$ of the TEIs were well matched in one- and two-level ACCF, respectively. The postoperative IBA and IBH values were consistent with the values of young individuals. The AA-TMC achieved good matching with cervical geometries and segmental alignment in one- and two-level ACCF, and is proposed for use in ACCF to increase the contact at the TEI and achieve sufficient lordosis restoration.
\end{abstract}

Correspondence to: Professor Xijing He, Department of Orthopedics, Second Affiliated Hospital of Xi'an Jiaotong University, 157 West 5 Road, Xi'an, Shaanxi 710004, P.R. China E-mail: he_xijing@126.com

Key words: cadaver, decompression, prosthesis design, spinal fusion

\section{Introduction}

Severe titanium mesh cage (TMC) subsidence $(>3 \mathrm{~mm})$ is the most common postoperative complication in anterior cervical corpectomy and fusion (ACCF) (1-7), which occurs in up to $30.8 \%$ of patients. Major loss of intervertebral body height $(\mathrm{IBH})$ notably alters cervical alignment, increases the stress load of fixation and decreases the volume of the spinal canal and intervertebral foramen $(4,7,8)$. These pathological changes can result in severe complications, such as kyphosis, nerve recompression and internal fixation failure, which greatly affect surgical outcomes (2-4,7).

The sharp footprints and flat end of conventional TMCs play important roles in subsidence (Figs. 1 and 2) $(2,4,7,9)$. These design defects lead to a very small contact area at the TMC-endplate interface (TEI), which results in high stress concentration and causes the TMC to perforate easily into the endplate $(4,7,10)$. To increase the contact area and thus homogeneously distribute the stress at the TEI (11-13), several cages with enlarged end surfaces have been applied in ACCF $(7,14,15)$. However, even if the end surface is enlarged, the rate of TMC subsidence is frequently still high at the last follow-up $(7,14,15)$, primarily because the ends of these devices are still flat, and the actual contact area at the TEI does not effectively increase $(4,10,16)$. Therefore, to increase the contact area and reduce the concentration of stress at the TEI, the geometries of the TMC ends should be designed such that they are adaptive to the endplate.

The aim of the present study was to design an optimal quadrate anatomically adaptive titanium mesh cage (AA-TMC) that achieves good geometrical matching with the TEI, IBH and intervertebral body angle (IBA) in oneand two-level ACCF. To ensure good geometrical matching, the end shape, height and diameter of the AA-TMC were identified based on the morphological measurements of cervical geometries. After being constructed, the AA-TMC was implanted into cervical cadaveric specimens and underwent computed tomography (CT) tests to further assess its effects on TEI matching and the maintenance of IBA and IBH. 


\section{Materials and methods}

Endplate geometry measurements. The present study was approved by the Ethics Committee of Xi'an Jiaotong University Second Affiliated Hospital (Xi'an, China). The cervical CT images of 71 individuals were obtained by searching the CT database in Xi'an Jiaotong University Second Affiliated Hospital (from April 2016 to July 2017). The images were imported into Mimics Innovation Suite 17 (Materialise NV) to measure the endplate geometries. Individuals with obvious degeneration, obvious osteophytes, fracture, infection or metastasis were excluded. Finally, a total of 54 subjects were included for data measurement (35 males and 19 females; average age, $56.54 \pm 11.34$ years; age range, $37-81$ years).

The interior endplates (IEPs) from $\mathrm{C} 3$ to $\mathrm{C} 6$ and the superior endplates (SEPs) from $\mathrm{C} 4$ to $\mathrm{C} 7$ were included for analysis. In total, five parameters were measured (Fig. 3): i) The middle sagittal anteroposterior diameter (APD); ii) the middle coronal transverse diameter (TD); iii) the middle sagittal radius of the endplate; iv) the middle coronal radius of the endplate; and v) the concave depth of the endplate. When measuring the endplate radius, if the shape of the endplate was not obviously arched, an arc was drawn through the deepest point of the endplate, as well as the end points of the APD or TD. This arc approximately and quantitatively described the shape of the endplate, which was considered arched when the concave depth was $>1 \mathrm{~mm}$. The concave depth was calculated as follows:

$$
\begin{aligned}
& \text { Sagittal concave depth }=\text { Radius }-\sqrt{\text { Radius }^{2}-\left\{\frac{\mathrm{APD}^{2}}{2}\right\}^{2}} \\
& \text { Coronal concave depth }=\text { Radius }-\sqrt{\text { Radius }^{2}-\left\{\frac{\mathrm{TD}^{2}}{2}\right\}^{2}}
\end{aligned}
$$

IBA and IBH measurements. The cervical X-rays of 88 young individuals were collected by searching the $\mathrm{X}$-ray database in Xi'an Jiaotong University Second Affiliated Hospital from March 2017 to July 2018, and were used to measure the IBA and IBH of the C4, C5, C6, C4-5 and C5-6 levels. The IBA of $\mathrm{C} 4$ referred to the angle between the $\mathrm{C} 3$ inferior endplate and $\mathrm{C} 5$ superior endplate. The IBH of $\mathrm{C} 4$ referred to the distance between the $\mathrm{C} 3$ inferior endplate and $\mathrm{C} 5$ superior endplate. The IBA of $\mathrm{C} 5$ referred to the angle between $\mathrm{C} 4$ inferior endplate and C6 superior endplate. The IBH of C5 indicated the distance between the $\mathrm{C} 4$ inferior endplate and $\mathrm{C} 6$ superior endplate. The IBA of $\mathrm{C} 6$ referred to the angle between the C5 inferior endplate and $\mathrm{C} 7$ superior endplate. The IBH of C6 referred to the distance between the $\mathrm{C} 5$ inferior endplate and C7 superior endplate. The IBA of C4-5 referred to the angle between the $\mathrm{C} 3$ inferior endplate and $\mathrm{C} 6$ superior endplate. The IBH of C4-5 referred to the distance between the $\mathrm{C} 3$ inferior endplate and C6 superior endplate. The IBA of C5-6 referred to the angle between the $\mathrm{C} 4$ inferior endplate and $\mathrm{C} 7$ superior endplate. The IBH of C5-6 referred to the distance between the $\mathrm{C} 4$ inferior endplate and $\mathrm{C} 7$ superior endplate. The measurement methods are presented in Fig. 3. Patients with cervical straightening, kyphosis, fracture, intervertebral space narrowing, obvious osteophytes, infection or metastasis were excluded. Finally, a total of 74 subjects were included for IBA and IBH measurements (44 males and 30 females; average age, $33.88 \pm 7.6$ years; age range, $27-77$ years).

Description of AA-TMC. The shape of the AA-TMC was designed based on the average values of the measured parameters (APD, TD, sagittal radius, coronal radius, IBA and IBH). Both ends of the AA-TMC were domed. In the side view, the TMC was designed to be curved, and the curve of the cage was determined by IBA and IBH. In the axial view, the AA-TMC was quadrate. A total of 12 AA-TMCs with different sizes were designed with the following dimensions (parameters, height $\mathrm{x}$ anteroposterior diameter $\mathrm{x}$ transversal diameter; Fig. 4): $23 \times 12 \times 12,25 \times 12 \times 12,27 \times 12 \times 12,23 \times 14 \times 14,25 \times 14 \times 14$, $27 \times 14 \times 14,40 \times 12 \times 12,43 \times 12 \times 12,46 \times 12 \times 12,40 \times 14 \times 14$, $43 \times 14 \times 14$ and $46 \times 14 \times 14 \mathrm{~mm}$. The surface aeras of the inferior and superior TMC ends were the $0.84 \mathrm{~cm}^{2}$ and $0.91 \mathrm{~cm}^{2}$, respectively. All AA-TMCs were constructed using a selective laser melting 3D-printing machine (BLT-S300; Xi'an Bright Additive Technologies Co., Ltd.).

Measurements of TEI matching degree, postoperative IBA and IBH. A total of 18 formalin-fixed cervical cadaveric specimens were obtained from the Department of Human Anatomy and Tissue Embryology of Xi'an Jiaotong University (11 males and 7 females; average age, $67.83 \pm 13.37$ years; age range, $42-85$ years). All of the specimens were examined by X-ray to exclude evident osteophytes, fracture, infection and metastasis. Written informed consent for the use of these tissues was obtained from relatives prior to sample collection. These specimens were randomly assigned to 3 groups ( $n=6 /$ group): C4 corpectomy, C5 corpectomy and C6 corpectomy. After one-level corpectomy, the surgeon chose a suitably sized AA-TMC and inserted it into the intervertebral body space. The specimen then underwent CT scanning $(120 \mathrm{kV}$; $200 \mathrm{~mA}$; slice thickness, $1.25 \mathrm{~mm}$ ). The images were imported into Mimics 17 to assess IBA, IBH and TEI matching. Subsequently, the six specimens in the $\mathrm{C} 5$ corpectomy group were randomly reassigned into the $\mathrm{C} 4-5$ corpectomy and the $\mathrm{C} 5-6$ corpectomy groups. The six specimens in the $\mathrm{C} 4$ corpectomy group were reassigned into the $\mathrm{C} 4-5$ corpectomy group and the six specimens in the $\mathrm{C} 6$ corpectomy group were reassigned into the C5-6 corpectomy group. The two-level ACCF surgical procedure and the same $\mathrm{CT}$ evaluations were then performed in each group. The evaluation of IBA, IBH and TEI matching was similar to that for one-level corpectomy.

TEI matching evaluation was performed in the middle sagittal and coronal planes of the endplate. The matching outcomes were classified into 3 grades: Grade I, maximum interval between the TMC and endplate $<0.5 \mathrm{~mm}$; grade II, maximum interval between $0.5-1 \mathrm{~mm}$; and grade III, maximum interval $>1 \mathrm{~mm}$.

Statistical analysis. All measurement data are presented as the mean \pm SD. SPSS 18.0 was used for statistical analysis. One-way analysis of variance was applied to compare the APD, TD, IBA, IBH, sagittal and coronal radii, and the sagittal and coronal concave depths among groups, with Bonferroni post hoc test subsequently used. Fisher's exact test was used to compare the arched rates among groups, with Bonferroni's correction applied when using multiple Fisher's exact tests for 


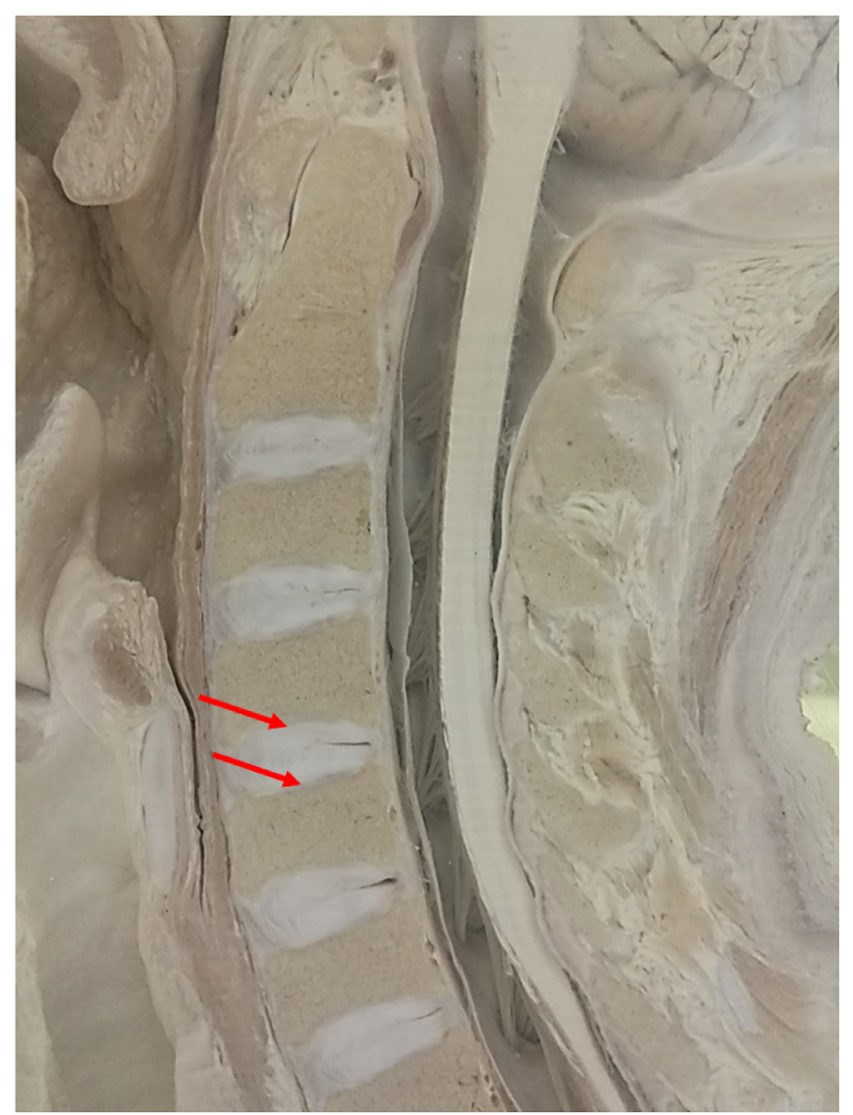

Figure 1. Middle sagittal image of a cadaveric cervical spine. The image shows that the inferior endplate is arched, and that the superior endplate is in an oblique position.

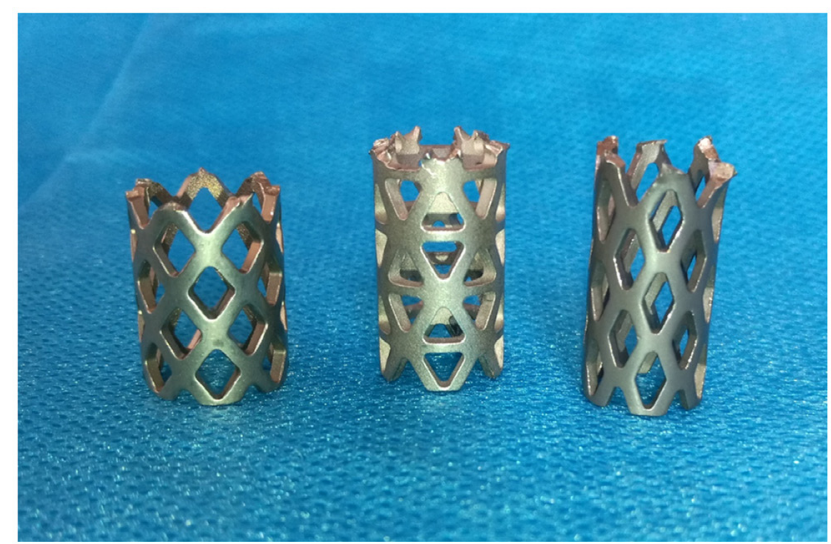

Figure 2. Shape of the conventional TMC. There are two shortcomings of the conventional TMC. First, both ends of the TMC are flat, which do not match well with the geometry of the endplate. Second, the TMC must be trimmed to a suitable length to fit the intervertebral body height, which leaves sharp footprints on the end. Both shortcomings lead to a very small contact area at the TMC-endplate interface. TMC, titanium mesh cage.

multiple comparisons. $\mathrm{P}<0.05$ was considered to indicate a statistically significant difference.

\section{Results}

Endplate geometries. The outcomes of the endplate geometries obtained from the patients' CT images are presented in Tables I and II. In general, the APDs and TDs of the IEP progressively increased from C3 IEP (16.20 \pm 1.81 and $15.89 \pm 1.69 \mathrm{~mm}$, respectively) to C6 IEP $(17.23 \pm 1.74$ and $21.46 \pm 2.29 \mathrm{~mm}$, respectively). The APDs and TDs of the SEP progressively increased from C4 SEP $(15.31 \pm 1.921$ and $14.30 \pm 1.55 \mathrm{~mm}$, respectively) to C7 SEP $(16.42 \pm 1.78$ and $18.54 \pm 2.04 \mathrm{~mm}$, respectively). The mean surface areas of the SEP and IEP were 2.56 and $3.04 \mathrm{~cm}^{2}$, respectively (mean ATP multiplied by mean TD). Regarding the sagittal radii of the endplate, the IEP increased from C3 IEP $(19.51 \pm 6.59 \mathrm{~mm})$ to C6 IEP $(24.21 \pm 9.33 \mathrm{~mm})$, and the SEP reduced from C4 SEP $(135.78 \pm 58.91 \mathrm{~mm})$ to $\mathrm{C} 7 \mathrm{SEP}(90.00 \pm 57.29 \mathrm{~mm})$. The coronal radii of the $\mathrm{C} 3$ and $\mathrm{C} 4$ IEPs were $113.6 \pm 57.09$ and $126.70 \pm 57.23 \mathrm{~mm}$, respectively, which were significantly higher than those of C5 and C6 (79.98 \pm 37.90 and $68.48 \pm 25.86 \mathrm{~mm}$, respectively). The coronal radius of C7 SEP was $101.33 \mathrm{~mm}$, which was significantly higher than those of the SEPs of C4, C5, and C6 $(87.18 \pm 66.34,73.56 \pm 41.25$, and $94.97 \pm 60.23 \mathrm{~mm}$, respectively). In addition, the mean depths of the IEPs in the sagittal and coronal planes were $1.80 \pm 0.61$ and $0.59 \pm 0.40 \mathrm{~mm}$, respectively. The mean depths of the SEPs in the sagittal and coronal planes were $0.39 \pm 0.29$ and $0.51 \pm 0.34 \mathrm{~mm}$, respectively. Most of the IEPs in the sagittal plane were arched (93.8\%), whereas the majority of the IEPs in the coronal plane, and the majority of the SEPs in the sagittal and coronal planes were flat $(82.9,94.8$ and $93.8 \%$, respectively).

IBA and IBH of young individuals. The mean IBH and IBA values after one-level ACCF were $23.90 \pm 2.18 \mathrm{~mm}$ and $11.62 \pm 2.67^{\circ}$, respectively. In addition, the mean IBH and IBA values after two-level ACCF were $42.93 \pm 3.51 \mathrm{~mm}$ and $15.63 \pm 5.06^{\circ}$, respectively. No statistically significant differences were observed between the included levels (Table III).

TEI matching degrees and postoperative IBA and IBH. The outcomes of the TEI matching classification are shown in Tables IV and V. For one-level ACCF, $91.7 \%$ of the TEIs were classified as grade I, and $3.8 \%$ of the TEIs were classified as grade II (Figs. 5A and B, and 6). In two-level ACCF, 94.4\% of the TEIs were classified as grade I (Fig. 5C and D), and $1.4 \%$ of the TEIs were classified as grade II. For both one- and two-level ACCF, 93.1\% of the TEIs were classified as grade I. A total of 2.7 and $4.2 \%$ of the TEIs were classified as grades II and III, respectively. After one-level ACCF, the mean IBH and IBA values were $24.24 \pm 1.15 \mathrm{~mm}$ and $12.24 \pm 0.65^{\circ}$, respectively. After two-level ACCF, the mean IBH and IBA values were $42.79 \pm 1.70 \mathrm{~mm}$ and $16.26 \pm 1.27^{\circ}$, respectively (Table VI). No statistically significant differences were found between the included levels for postoperative IBA or IBH.

\section{Discussion}

The present study showed that $93.1 \%$ of the TEIs were $<0.5 \mathrm{~mm}$ (grade I). This proximity at the TEI ensured a maximum increase in contact and a homogeneous stress distribution at the TEI, which is beneficial to subsidence resistance. Additionally, with the use of the AA-TMC, IBA and IBH were successfully reconstructed in one- and two-level ACCF, suggesting that the AA-TMC may help to achieve 


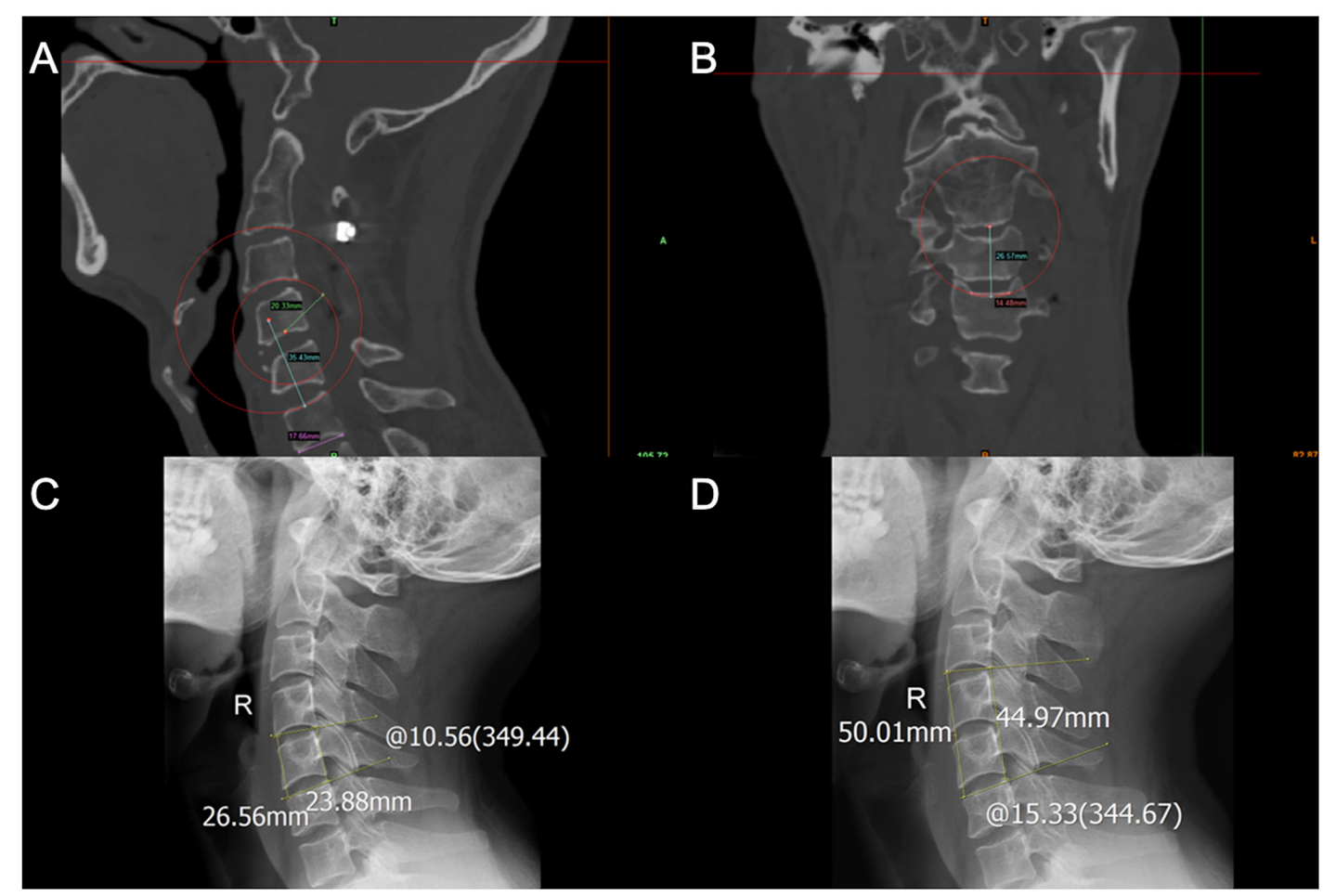

Figure 3. Parameter measurements from the computed tomography and X-ray images. (A) Measurement of the sagittal radius of C3 IEP (green), the sagittal radius of C6 SEP (blue) and the APD of C6 IEP (purple). (B) Measurement of the coronal radius and TD of C4 SEP. (C) Measurement of the IBA anterior IBH and posterior IBH of C5 corpectomy. The values shown on this figure after the symbol ' @' are degrees ( ${ }^{\circ}$ ). (D) Measurement of the IBA, anterior SSH and posterior SSH of C4-5 corpectomy. IBA, intervertebral body angle. The values shown on this figure after the symbol '@' are degrees $\left({ }^{\circ}\right)$; IBH, intervertebral body height; IEP, interior endplate; SEP, superior endplate; R, X-ray image shot from the right lateral position.

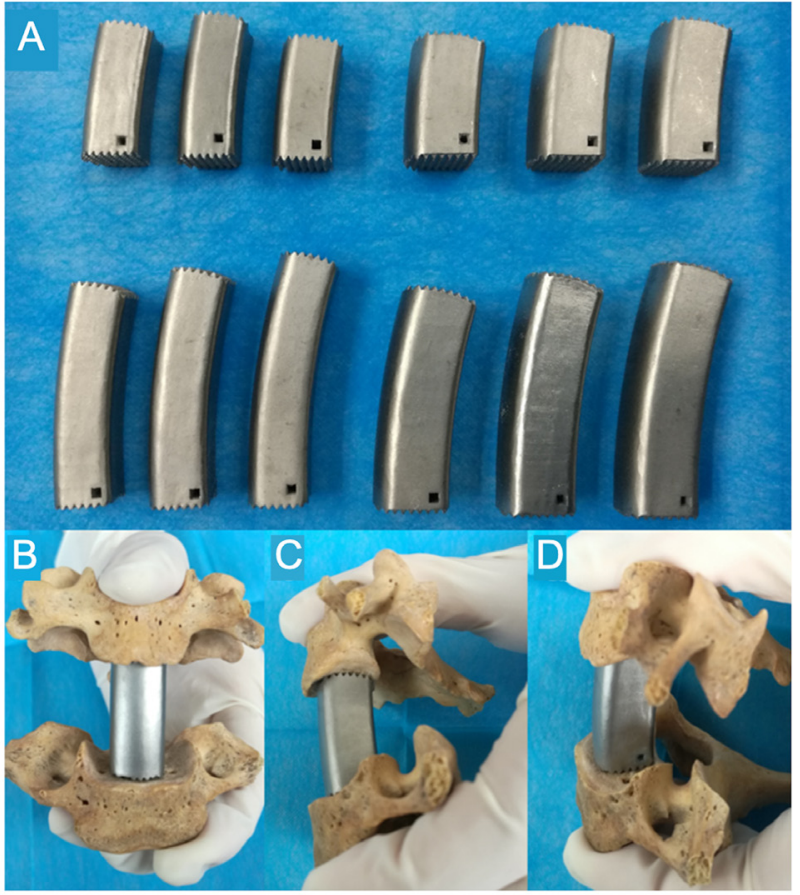

Figure 4. Overview of TMC. (A) A total of 12 TMCs with different lengths and diameters were constructed. (B-D) The TMC can be well matched with the endplates. (B) Front view, (C) IEP view and (D) SEP view. TMC, titanium mesh cage.

sufficient neural decompression and lordosis at the surgical level. Furthermore, the new AA-TMC was designed with a quadrate shape, which is considered to be advantageous over the conventional cylindrical TMC $(9,12,17,18)$. First, a quadrate-shaped AA-TMC has a greater contact area with the posterior part of the endplate, increasing the mechanical strength at the TEI $(12,17)$. Second, the quadrate AA-TMC has a larger volume for bone grafting $(9,18)$. Last, both sides of the AA-TMC are flat, which significantly increases the contact area with the remaining vertebral body and facilitates bony fusion $(9,18)$.

Various morphological studies, as well as the present study, have revealed that the cervical endplate is not simply flat (10,19-21); rather, most IEPs are arched (10,21). Although most SEPs are considered relatively flat, they still exhibit a concave depth of 0.39-0.69 $\mathrm{mm}(19,20)$. Therefore, the flat end of conventional TMCs do not match well with the endplate, leading to a very small contact area and high stress concentration at the TEI $(4,7,10)$. Therefore, the ends of the AA-TMC were designed based on the mean values of the endplate shape parameters to optimize the contact area at the TEI. The results showed that a good matching rate at the TEI was successfully obtained for the AA-TMC, and $93.1 \%$ of the new TMC made close contact with the endplate $(<0.5 \mathrm{~mm})$, thus alleviating the stress concentration at the TEI and effectively resisting subsidence. However, $4.2 \%$ of the TEIs still exhibited a large interval ( $>1 \mathrm{~mm}$, grade III); thus, their mechanical strength may decrease. In some cases, mismatch is inevitable; matching $100 \%$ of the endplates for a fixed-shape AA-TMC is impossible due to the large variations in endplate radius, concave depth and concave apex location among cervical endplates $(10,19-21)$. 
Table I. Middle sagittal plane endplate shape parameters.

\begin{tabular}{lcllr}
\hline A, IEP & & & & \\
\hline Level & APD, mm & Radius, mm & Depth, mm & Arched/Total $(\%)$ \\
\hline C3 $(n=44)$ & $16.20 \pm 1.81^{\mathrm{a}, \mathrm{b}}$ & $19.51 \pm 6.59^{\mathrm{c}, \mathrm{d}}$ & $1.91 \pm 0.51$ & $44 / 44(100)^{\mathrm{i}, \mathrm{j}}$ \\
C4 $(\mathrm{n}=53)$ & $16.65 \pm 2.18$ & $21.06 \pm 7.97^{\mathrm{e}}$ & $1.88 \pm 0.54$ & $52 / 53(98.1)^{\mathrm{k}}$ \\
C5 $(\mathrm{n}=48)$ & $17.10 \pm 2.19$ & $27.40 \pm 11.04$ & $1.58 \pm 0.66^{\mathrm{f}-\mathrm{h}}$ & $40 / 48(80)$ \\
C6 $(\mathrm{n}=48)$ & $17.23 \pm 1.74$ & $24.21 \pm 9.33$ & $1.82 \pm 0.67$ & $43 / 48(89.6)$ \\
Total $(\mathrm{n}=193)$ & $16.8 \pm 2.02$ & $23.07 \pm 9.34$ & $1.80 \pm 0.61$ & $181 / 193(93.8)$ \\
\hline
\end{tabular}

$\mathrm{B}, \mathrm{SEP}$

\begin{tabular}{lcccc}
\hline Level & APD, mm & Radius, mm & Depth, mm & Arched/Total $(\%)$ \\
\hline C4 $(\mathrm{n}=51)$ & $15.31 \pm 1.92^{1-\mathrm{n}}$ & $135.78 \pm 58.91$ & $0.27 \pm 0.14^{\mathrm{r}, \mathrm{s}}$ & $0 / 51(0)$ \\
C5 $(\mathrm{n}=54)$ & $16.14 \pm 2.26$ & $133.11 \pm 74.83$ & $0.35 \pm 0.3^{\mathrm{t}}$ & $2 / 54(3.6)$ \\
C6 $(\mathrm{n}=48)$ & $16.49 \pm 2.22$ & $115.63 \pm 70.71$ & $0.45 \pm 0.35$ & $1 / 48(2.1)$ \\
C7 $(\mathrm{n}=40)$ & $16.42 \pm 1.78$ & $90.00 \pm 57.29^{\mathrm{o}-\mathrm{q}}$ & $0.51 \pm 0.30$ & $4 / 40(10)$ \\
Total $(\mathrm{n}=193)$ & $16.06 \pm 2.11$ & $120.53 \pm 68.17$ & $0.39 \pm 0.29$ & $10 / 193(5.2)$ \\
\hline
\end{tabular}

${ }^{\mathrm{a}} \mathrm{P}<0.05$ vs. $\mathrm{C} 5$ IEP; ${ }^{\mathrm{P}} \mathrm{P}<0.05$ vs. C6 IEP; ${ }^{\mathrm{c}} \mathrm{P}<0.05$ vs. C5 IEP; ${ }^{\mathrm{d}} \mathrm{P}<0.05$ vs. C6 IEP; ${ }^{\mathrm{e}}<0.05$ vs. C5 IEP; ${ }^{\text {f }} \mathrm{P}<0.05$ vs. C3 IEP, ${ }^{\mathrm{g}} \mathrm{P}<0.05$ vs. C4 IEP; ${ }^{h} \mathrm{P}<0.05$ vs. C6 IEP; ${ }^{\mathrm{P}}<0.05$ vs. C5 IEP; ${ }^{\mathrm{P}}<0.05$ vs. C6 IEP; ${ }^{\mathrm{k}} \mathrm{P}<0.05$ vs. C5 IEP; ${ }^{1} \mathrm{P}<0.05$ vs. C5 SEP; ${ }^{\mathrm{m}} \mathrm{P}<0.05$ vs. C6 SEP; ${ }^{\mathrm{N}}<0.05$ vs. C7 SEP; ${ }^{\circ} \mathrm{P}<0.05$ vs. C5 SEP; ${ }^{\mathrm{P} P}<0.05$ vs. C6 SEP; ${ }^{\mathrm{P}}<<0.05$ vs. C7 SEP; ${ }^{\mathrm{P}}<0.05$ vs. C6 SEP; ${ }^{\mathrm{s}} \mathrm{P}<0.05$ vs. C7 SEP; ${ }^{\mathrm{t}} \mathrm{P}<0.05$ vs. C7 SEP. APD, anteroposterior diameter; IEP, inferior endplate; SEP, superior endplate.

Table II. Middle coronal plane endplate shape parameters.

\begin{tabular}{lcccc}
\hline A, IEP & & & & \\
\hline Level & TD, mm & Radius, mm & Depth, mm & Arched/Total $(\%)$ \\
\hline C3 $(n=44)$ & $15.89 \pm 1.69^{\mathrm{a}}$ & $113.6 \pm 57.09^{\mathrm{b}, \mathrm{c}}$ & $0.37 \pm 0.23^{\mathrm{d}}$ & $2 / 44(4.5)$ \\
C4 $(\mathrm{n}=53)$ & $17.00 \pm 1.86^{\mathrm{a}}$ & $126.70 \pm 57.23^{\mathrm{b}, \mathrm{c}}$ & $0.39 \pm 0.33^{\mathrm{e}}$ & $4 / 53(7.5)$ \\
C5 $(\mathrm{n}=48)$ & $17.94 \pm 2.22^{\mathrm{a}}$ & $79.98 \pm 37.90$ & $0.64 \pm 0.37^{\mathrm{f}-\mathrm{h}}$ & $6 / 48(12.5)$ \\
C6 $(\mathrm{n}=48)$ & $21.46 \pm 2.29^{\mathrm{a}}$ & $68.48 \pm 25.86$ & $0.95 \pm 0.33$ & $21 / 48(43.75)^{\mathrm{i}-\mathrm{k}}$ \\
Total $(\mathrm{n}=193)$ & $18.09 \pm 2.89$ & $97.61 \pm 52.1$ & $0.59 \pm 0.40$ & $33 / 193(17.1)$ \\
\hline
\end{tabular}

$\mathrm{B}, \mathrm{SEP}$

\begin{tabular}{lcccr}
\hline Level & TD, mm & Radius, mm & Depth, mm & Arched/Total $(\%)$ \\
\hline C4 $(\mathrm{n}=51)$ & $14.30 \pm 1.55^{1}$ & $87.18 \pm 66.34$ & $0.48 \pm 0.33$ & $4 / 51(7.8)$ \\
C5 $(\mathrm{n}=54)$ & $14.81 \pm 1.70^{\mathrm{m}}$ & $73.56 \pm 41.25^{\mathrm{q}, \mathrm{r}}$ & $0.48 \pm 0.30$ & $1 / 54(1.8)^{\mathrm{s}}$ \\
C6 $(\mathrm{n}=48)$ & $16.82 \pm 2.50$ & $94.97 \pm 60.23$ & $0.51 \pm 0.28$ & $3 / 48(6.3)$ \\
C7 $(\mathrm{n}=40)$ & $18.54 \pm 2.04^{\mathrm{n}-\mathrm{p}}$ & $101.33 \pm 51.70$ & $0.59 \pm 0.44$ & $6 / 40(15)$ \\
Total & $15.94 \pm 2.55$ & $88.22 \pm 56.17$ & $0.51 \pm 0.34$ & $12 / 193(6.2)$ \\
\hline
\end{tabular}

${ }^{a} \mathrm{P}<0.05$ for all the pairwise comparisons (C3 vs. C4, C3 vs. C5, C3 vs. C6, C4 vs. C5, C4 vs. C6, C5 vs. C6); ${ }^{\mathrm{P}}<0.05$ vs. C5 IEP; ${ }^{\mathrm{c}} \mathrm{P}<0.05$ vs. C6; ${ }^{\mathrm{d}} \mathrm{P}<0.05$ vs. C6 IEP; ${ }^{\mathrm{e}}<0.05$ vs. C6 IEP; ${ }^{\mathrm{P}}<0.05$ vs. C3 IEP; ${ }^{\mathrm{P}}<0.05$ vs. C4 IEP; ${ }^{\mathrm{P}}<0.05$ vs. C5 IEP,C4 and C5 IEPs; ${ }^{\mathrm{i}} \mathrm{P}<0.05$ vs. C3 IEP, ${ }^{\mathrm{j}} \mathrm{P}<0.05$ vs. C4 IEP; ${ }^{k} \mathrm{P}<0.05$ vs. C5 IEP; ${ }^{1} \mathrm{P}<0.05$ vs. C6 SEP; ${ }^{\mathrm{m}} \mathrm{P}<0.05$ vs. C6 SEP; ${ }^{\mathrm{P}} \mathrm{P}<0.05$ vs. $\mathrm{C} 4 \mathrm{SEP} ;{ }^{\circ} \mathrm{P}<0.05$ vs. C5 SEP; ${ }^{p} \mathrm{P}<0.05$ vs. C6 $\mathrm{SEP}$; ${ }^{\mathrm{P}}<0.05$ vs. C6 SEP; ${ }^{\mathrm{r}} \mathrm{P}<0.05$ vs. C7 SEP; ${ }^{\mathrm{P}}<0.05$ vs. C7 SEP. TD, transverse diameter; IEP, inferior endplate; SEP, superior endplate.

TEI matching is classified based on the thickness of the endplate, which ranges from 0.65 to $1.35 \mathrm{~mm}(22,23)$.
A grade I TEI allows the footprints that initially contact the endplate to subside by only $0.5 \mathrm{~mm}$, which is $\sim 50 \%$ of the 
Table III. Middle coronal plane IBH and IBA.

A, One level

\begin{tabular}{lcc}
\hline Level & IBH, mm & IBA, \\
\hline C4 $(n=74)$ & $24.15 \pm 2.32$ & $11.67 \pm 2.67$ \\
C5 $(n=74)$ & $23.72 \pm 2.11$ & $11.40 \pm 2.82$ \\
C6 $(n=72)$ & $23.83 \pm 2.12$ & $11.78 \pm 2.54$ \\
Total & $23.90 \pm 2.18$ & $11.62 \pm 2.67$ \\
\hline
\end{tabular}

B, Two level

\begin{tabular}{lcc}
\hline Level & IBH, mm & IBA, \\
\hline C4-5 $(\mathrm{n}=74)$ & $42.85 \pm 3.86$ & $15.80 \pm 5.05$ \\
C5-6 $(\mathrm{n}=72)$ & $43.01 \pm 3.14$ & $15.42 \pm 5.09$ \\
Total & $42.93 \pm 3.51$ & $15.63 \pm 5.06$ \\
\hline
\end{tabular}

All analyses were $\mathrm{P}>0.05$. IBH, intervertebral body height; IBA, intervertebral body angle.

Table IV. Titanium mesh cage-endplate matching classification (one-level anterior cervical corpectomy and fusion).

\begin{tabular}{lcc}
\hline A, Sagittal plane & & \\
\hline Grade & IEP, n (\%) & SEP, n (\%) \\
\hline I & $15(83.3)$ & $17(94.4)$ \\
II & $1(5.6)$ & $1(5.6)$ \\
III & $2(11.1)$ & $0(0)$ \\
\hline
\end{tabular}

$\mathrm{B}$, Coronal plane

\begin{tabular}{lcc}
\hline Grade & IEP, n (\%) & SEP, n (\%) \\
\hline I & $17(94.4)$ & $17(94.4)$ \\
II & $0(0)$ & $1(5.6)$ \\
III & $1(5.6)$ & $0(0)$ \\
\hline
\end{tabular}

IEP, inferior endplate; SEP, superior endplate.

endplate thickness; as such, the integrity of the endplate is not severely damaged $(22,23)$. Additionally, due to slight subsidence, the interval disappears, which results in a significant increase in the contact area and further alleviates the stress concentration at the interface (11-13). However, if the interval exceeds $1 \mathrm{~mm}$, the footprints that initially contact the endplate may perforate into the cancellous bone before the interval disappears, severely damaging the mechanical strength of the endplate (24).

In the present study, analysis of historical CT images was performed to measure endplate geometries, whereas X-ray images were used to measure IBA and IBH; there were several reasons for these decisions. First, measuring these parameters
Table V. Titanium mesh cage-endplate matching classification (two-level anterior cervical corpectomy and fusion).

\begin{tabular}{lcc}
\hline A, Sagittal plane & & \\
\hline Grade & IEP (\%) & SEP $(\%)$ \\
\hline I & $16(88.9)$ & $18(100)$ \\
II & $0(0)$ & $0(0)$ \\
III & $2(11.1)$ & $0(0)$ \\
\hline B, Coronal plane & & \\
\hline Grade & IEP $(\%)$ & SEP $(\%)$ \\
\hline I & $17(94.4)$ & $17(94.4)$ \\
II & $0(0)$ & $1(5.6)$ \\
III & $1(5.6)$ & $0(0)$
\end{tabular}

IEP, inferior endplate; SEP, superior endplate.

Table VI. Postoperative middle coronal plane IBH and IBA.

A, One-level ACCF

\begin{tabular}{lcc}
\hline Level & IBH, mm & IBA, \\
\hline C4 $(n=6)$ & $24.36 \pm 1.30$ & $12.25 \pm 0.89$ \\
C5 $(n=6)$ & $24.12 \pm 1.05$ & $12.31 \pm 0.59$ \\
C6 $(n=6)$ & $24.25 \pm 1.29$ & $12.17 \pm 0.52$ \\
Total & $24.24 \pm 1.15$ & $12.24 \pm 0.65$ \\
\hline
\end{tabular}

B, Two-level ACCF

\begin{tabular}{lcc}
\hline Level & IBH, mm & IBA, \\
\hline C4-5 $(n=9)$ & $43.12 \pm 1.81$ & $16.37 \pm 1.47$ \\
C5-6 $(n=9)$ & $42.46 \pm 1.63$ & $16.15 \pm 1.12$ \\
Total & $42.79 \pm 1.70$ & $16.26 \pm 1.27$
\end{tabular}

All analyses were $\mathrm{P}>0.05$. ACCF, anterior cervical corpectomy and fusion; IBH, intervertebral body height; IBA, intervertebral body angle.

using the CT images was more practical than using the X-ray images, particularly for the TD. Second, a previous study indicated that the depth of the endplate gradually increases with age (25). Endplate geometries in the elderly may be slightly different from those in younger populations. The CT images in the present study were obtained from elderly patients, whereas the X-ray images were obtained from young individuals; as ACCF is more frequently performed in the elderly (7-9), CT images were used to measure APD and TD to fit the endplate geometries of elderly patients. In addition, the cervical segmental alignment in young individuals was straighter compared with that in elderly patients. Therefore, 


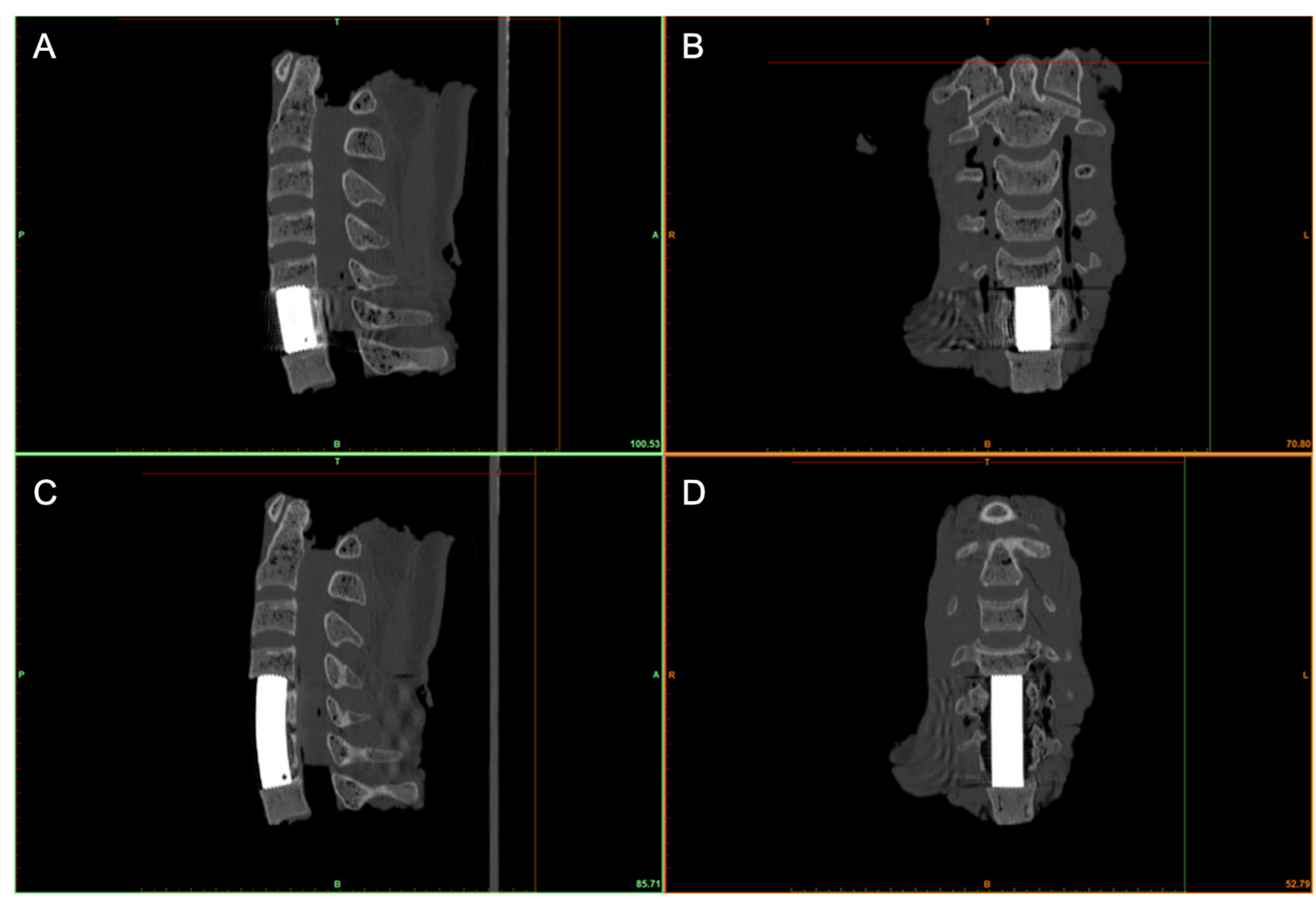

Figure 5. TEI matchings of grade I. Computed tomography images of (A and B) one- and (C and D) two-level anterior cervical corpectomy and fusion using the new AA-TMC. Both ends of the AA-TMC achieved good matching with the endplates in the (A and C) sagittal and (B and D) coronal planes. (A-D) The intervals at the TMC-endplate interface were classified as grade I. AA-TMC, anatomically adaptive titanium mesh cage.

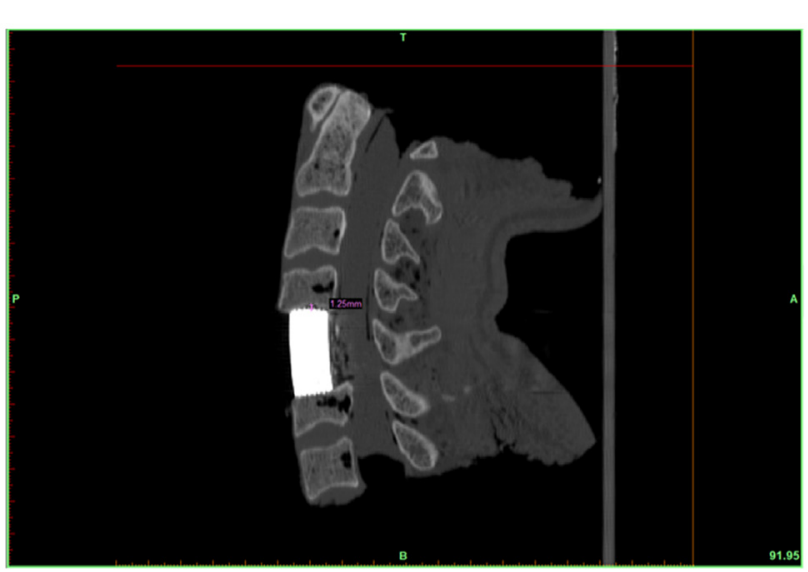

Figure 6. TEI matching of grade III. One case had an obvious interval at the TEI $(1.25 \mathrm{~mm})$ and was classified as grade III.

IBA and IBH were measured in the X-ray images of young individuals.

In addition to simulating the endplate shape, simulating the IBA of the surgical segment in the TMC design also determines the degree of TEI matching $(2,9,18)$. Due to the IBA, the SEP is in an oblique position, which causes conventional TMCs to contact the SEP at the posterior region $(4,6,7)$. This geometric mismatch produces a high stress concentration at the posterior region and causes most of the subsidence to occur at the posterior-inferior part of the TMC $(4,6)$. Therefore, the
IBA must be simulated in the TMC design to ensure good TEI matching. Our previous study reported that simulating the IBA in the TMC can improve the maximum load of the endplate by $32.6 \%$ compared with in conventional TMCs, thus effectively improving the subsidence resistance (18). In the present study, the AA-TMC was designed with a curved shape to simulate the IBA of the surgical segments and to ensure good matching at the TEI. Additionally, the curved AA-TMC can be aligned with the cervical lordosis, ensuring that the AA-TMC is located inside the residual vertebral body after multilevel corpectomy.

Simulating the IBA in an AA-TMC can successfully maintain cervical lordosis $(2,9)$. Although using conventional TMCs can achieve normal IBA by the final follow-up, the IBA was significantly reduced compared with the IBA immediately after surgery $(2,6)$. Restoration of a normal IBA depends on the TMC subsidence at the posterior-inferior region $(2,6)$. In contrast, by using an AA-TMC, the IBA of the surgical segment can be restored to normal immediately after surgery $(2,9)$. Additionally, due to the effective increase in the TEI contact area, the IBH of the surgical segment can be well maintained in the long term $(2,9)$. In the present study, postoperative IBA and IBH values using cadaveric specimens were consistent with the values of young individuals. Thus, combined with the advantage of subsidence resistance, the new AA-TMC reported in the present study may ensure that the surgical segment achieves sufficient neural decompression and maintains cervical lordosis at all times. 
Increasing the end surface of the TMC is also beneficial for preventing subsidence by helping to distribute the stress homogeneously from the cranial region to the endplate (11-13). However, over-enlarging the end surface may impact the fusion rate (26). Thus, bony fusion and mechanical strength must be balanced to achieve optimal outcomes for both (26). Previous biomechanics studies revealed that when the implant end reaches $30 \%$ of the endplate surface area, the maximum compressive load at the interface is similar to that at an implant end with a much larger surface area $(18,26)$. As presented in the results, the mean surface areas of the SEP and IEP were 2.56 and $3.04 \mathrm{~cm}^{2}$. Correspondingly, $\sim 0.84$ and $\sim 0.91 \mathrm{~cm}^{2}$ of the surface areas of the inferior and superior TMC ends, respectively, could produce a sufficient compressive load to resist subsidence and leave sufficient space for bony fusion. In the present study, only an outline of the TMC was designed and constructed, as the primary aim of the study was to assess the effects of the new TMC on TEI matching, and restoration of segmental IBA and IBH. Thus, the TMC should be further developed to include an appropriate autograft space for clinical use.

The present study has several limitations. First, the sample sizes of the cadaveric specimens in each one-level corpectomy group were small. A small sample size may result in selection bias and affect the measurement results of the matching degree, IBA and IBH. Second, further biomechanical studies using the AA-TMC for vertebral body reconstruction in ACCF should be performed to assess the stability and durability of the surgical construct in detail.

The new quadrate AA-TMC, which was designed based on cervical anatomical parameters, successfully matched well with the endplate, and restored the normal IBA and IBH; thus, this new AA-TMC may be beneficial for preventing subsidence, and maintaining sufficient neural decompression and lordosis. Due to these advantages, the new TMC is advocated for use in ACCF to include an autograft space after further development.

\section{Acknowledgements}

The authors would like to thank Dr Quanxin Yang and Dr Xiaohui Li (both Department of Radiology, Second Affiliated Hospital of Xi'an Jiaotong University) for their help in the CT and X-ray data search. The authors would also like to thank Dr Xiaoqian Zhou (Department of Radiology, Second Affiliated Hospital of Xi'an Jiaotong University) for her help with the CT scans of the cadaveric cervical spine specimens.

\section{Funding}

This work was supported by the National Natural Science Foundation of China (grant no. 81571209). No benefits in any form were received from a commercial party during the course of this work.

\section{Availability of data and materials}

All data generated or analyzed during this study are included in this published article.

\section{Authors' contributions}

TL wrote the paper and designed the study as well as performed the ACCF surgery and data collection. YW performed the ACCF surgery and data collection. ZG and JL examined the $\mathrm{CT}$ and X-ray images and performed the image measurements. NL and CL performed the statistical analysis. XH designed and supervised the study. TL, YW, ZG, JL, NL, CL and XH confirm the authenticity of all the raw data. All authors have read and approved the final manuscript.

\section{Ethics approval and consent to participate}

The present study was approved by the Ethics Committee of Xi'an Jiaotong University Second Affiliated Hospital. Written informed consent for the use of cadaveric specimens were obtained from relatives prior to sample collection.

\section{Patient consent for publication}

Not applicable.

\section{Competing interests}

The authors declare that they have no competing interests.

\section{References}

1. Zeng J, Duan Y, Yang Y, Wang B, Hong Y, Lou J, Ning N and Liu H: Anterior corpectomy and reconstruction using dynamic cervical plate and titanium mesh cage for cervical spondylotic myelopathy: A minimum 5-year follow-up study. Medicine (Baltimore) 97: e9724, 2018.

2. Fengbin Y, Jinhao M, Xinyuan L, Xinwei W, Yu C and Deyu C: Evaluation of a new type of titanium mesh cage versus the traditional titanium mesh cage for single-level, anterior cervical corpectomy and fusion. Eur Spine J 22: 2891-2896, 2013.

3. Yan D, Wang Z, Deng S, Li J and Soo C: Anterior corpectomy and reconstruction with titanium mesh cage and dynamic cervical plate for cervical spondylotic myelopathy in elderly osteoporosis patients. Arch Orthop Trauma Surg 131: 1369-1374, 2011

4. Wu J, Luo D, Ye X, Luo X, Yan L and Qian H: Anatomy-related risk factors for the subsidence of titanium mesh cage in cervical reconstruction after one-level corpectomy. Int J Clin Exp Med 8: 7405-7411, 2015.

5. Weber MH, Fortin M, Shen J, Tay B, Hu SS, Berven S, Burch S, Chou D, Ames C and Deviren V: Graft subsidence and revision rates following anterior cervical corpectomy: A clinical study comparing different interbody cages. Clin Spine Surg 30: E1239-E1245, 2017.

6. Jang JW, Lee JK, Lee JH, Hur H, Kim TW and Kim SH: Effect of posterior subsidence on cervical alignment after anterior cervical corpectomy and reconstruction using titanium mesh cages in degenerative cervical disease. J Clin Neurosci 21: 1779-1785, 2014.

7. Chen Y, Chen D, Guo Y, Wang X, Lu X, He Z and Yuan W: Subsidence of titanium mesh cage: A study based on 300 cases. J Spinal Disord Tech 21: 489-492, 2008.

8. Hartmann S, Kavakebi P, Wipplinger C, Tschugg A, Girod PP, Lener S and Thomé C: Retrospective analysis of cervical corpectomies: Implant-related complications of one- and two-level corpectomies in 45 patients. Neurosurg Rev 41: 285-290, 2018.

9. Lu T, Liu C, Yang B, Liu J, Zhang F, Wang D, Li H and He X: Single-level anterior cervical corpectomy and fusion using a new 3D-printed anatomy-adaptive titanium mesh cage for treatment of cervical spondylotic myelopathy and ossification of the posterior longitudinal ligament: A retrospective case series study. Med Sci Monit 23: 3105-3114, 2017. 
10. Lou J, Liu H, Rong X, Li H, Wang B and Gong Q: Geometry of inferior endplates of the cervical spine. Clin Neurol Neurosurg 142: 132-136, 2016

11. Ordway NR, Rim BC, Tan R, Hickman R and Fayyazi AH: Anterior cervical interbody constructs: Effect of a repetitive compressive force on the endplate. J Orthop Res 30: 587-592, 2012.

12. Lowe TG, Hashim S, Wilson LA, O'Brien MF, Smith DA, Diekmann MJ and Trommeter J: A biomechanical study of regional endplate strength and cage morphology as it relates to structural interbody support. Spine (Phila Pa 1976) 29: 2389-2394, 2004.

13. Hasegawa K, Abe M, Washio T and Hara T: An experimental study on the interface strength between titanium mesh cage and vertebra in reference to vertebral bone mineral density. Spine (Phila Pa 1976) 26: 957-963, 2001.

14. Brenke C, Fischer S, Carolus A, Schmieder K and Ening G: Complications associated with cervical vertebral body replacement with expandable titanium cages. J Clin Neurosci 32: 35-40, 2016.

15. Tarantino R, Nigro L, Donnarumma P, Rullo M, Santoro A and Delfini R: Cervical reconstruction techniques. After adequate selection of the patient report of a series of 34 patients treated with winged expandable cages. Neurosurg Rev 40: 281-286, 2017.

16. Chen H, Zhong J, Tan J, Wu D and Jiang D: Sagittal geometry of the middle and lower cervical endplates. Eur Spine J 22: 1570-1575, 2013

17. Ordway NR, Lu YM, Zhang X, Cheng CC, Fang $\mathrm{H}$ and Fayyazi AH: Correlation of cervical endplate strength with CT measured subchondral bone density. Eur Spine J 16: 2104-2109, 2007.

18. Lu T, Liang H, Liu C, Guo S, Zhang T, Yang B and He X: Effects of titanium mesh cage end structures on the compressive load at the endplate interface: A cadaveric biomechanical study. Med Sci Monit 23: 2863-2870, 2017.
19. Feng H, Fang X, Huang D, Yu C, Zhao S and Hao D: Quantitative morphometric study of the subaxial cervical vertebrae end plate. Spine J 17: 269-276, 2017.

20. Feng H, Fang XY, Huang DG, Yu CC, Li HK, Zhao SC, Ge CY, Bai RH and Hao DJ: A morphometric study of the middle and lower cervical vertebral endplates and their components. Medicine (Baltimore) 96: e6296, 2017.

21. Zhao S, Hao D, Jiang Y, Huang D, Ge C and Feng $H$ Morphological studies of cartilage endplates in subaxial cervical region. Eur Spine J 25: 2218-2222, 2016.

22. Schmitz B, Pitzen T, Beuter T, Steudel WI and Reith W: Regional variations in the thickness of cervical spine endplates as measured by computed tomography. Acta Radiol 45: 53-58, 2004

23. Pitzen T, Schmitz B, Georg T, Barbier D, Beuter T, Steudel WI and Reith W: Variation of endplate thickness in the cervical spine. Eur Spine J 13: 235-240, 2004.

24. Cheng CC, Ordway NR, Zhang X, Lu YM, Fang H and Fayyazi AH: Loss of cervical endplate integrity following minimal surface preparation. Spine (Phila Pa 1976) 32: 1852-1855, 2007

25. van der Houwen EB, Baron P, Veldhuizen AG, Burgerhof JG, van Ooijen PM and Verkerke GJ: Geometry of the intervertebral volume and vertebral endplates of the human spine. Ann Biomed Eng 38: 33-40, 2010

26. Steffen T, Tsantrizos A and Aebi M: Effect of implant design and endplate preparation on the compressive strength of interbody fusion constructs. Spine (Phila Pa 1976) 25: 1077-1084, 2000.

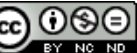

This work is licensed under a Creative Commons Attribution-NonCommercial-NoDerivatives 4.0 International (CC BY-NC-ND 4.0) License. 